\title{
元素时空博物馆一一成人之 “镁”
}

李宇琛 ${ }^{1}$, 牛嘉平 2 , 关吴 ${ }^{2}$, 钟丰璘 ${ }^{2}$, 颜静 1 , ${ }^{*}$, 间毅 $1,2,{ }^{*}$

1 西北工业大学化学与化工学院, 西安 710129

2 西北工业大学伦敦玛丽女王大学工程学院, 西安 710072

摘要: 元素时空博物馆举办了 “镁” 的专场活动, 馆长张博士和五位嘉宾分别从镁元素的发现、镁的燃烧、镁合金、 格氏试剂以及镁在生物医学方面的应用等视角向参观者介绍了镁这种元素。

关键词: 镁; 格氏试剂

中图分类号: G64; O6

\section{Museum of Element Time and Space: A Tale of Magnesium}

\author{
Yuchen $\mathrm{Li}^{1}$, Jiaping Niu ${ }^{2}$, Hao Guan ${ }^{2}$, Fenglin Zhong ${ }^{2}$, Jing Yan ${ }^{1, *}$, Yi Yan 1,2,* \\ ${ }^{1}$ School of Chemistry and Chemical Engineering, Northwestern Polytechnical University, Xi'an 710129, P. R. China. \\ ${ }^{2}$ Queen Mary University of London Engineering School, Northwestern Polytechnical University, Xi'an 710072, P. R. China.
}

Abstract: The Museum of element time and space held a special activity of "magnesium". Curator Dr. Zhang and five guests introduced magnesium to visitors from the perspectives of discovery, phenomena of its combustion, alloy, Grignard reagent and applications of magnesium in biomedicine.

Key Words: Magnesium; Grignard reagent

元素时空博物馆 “前世今生”活动项目今天迎来了镁元素的专场，希望一中的同学们前去参观， 受到了张馆长的热烈欢迎。

张馆长笑眯眯地对同学们讲道: “今天我们将通过 $3 \mathrm{D}$ 立体影像技术请来五位嘉宾, 带大家全面 地认识 “镁” 这个我们日常生活中的 “老熟人’, 让我们一起看看它有哪些鲜为人知的小秘密吧! 首 先我给大家简单介绍一下: 镁, 元素周期表中第 12 号元素, 位于 $s$ 区第二主族, 是典型的二价金属 元素。纯净的镁单质呈银白色, 带有金属光泽, 而我们日常生活中见到的镁由于在空气中氧化而略 显灰白。镁的密度很小, 延展性、导电性和导热性都很好, 是最轻的有用金属之一。在地壳中, 镁 以菱镁矿 $\left(\mathrm{MgCO}_{3}\right)$ 、白云石 $\left(\mathrm{CaMg}\left(\mathrm{CO}_{3}\right)_{2}\right)$ 、光卤石 $\left(\mathrm{KCl} \cdot \mathrm{MgCl}_{2} \cdot 6 \mathrm{H}_{2} \mathrm{O}\right)$ 等形式广泛存在，含量高达 $2 \%$, 算得上地壳中最丰富的元素之一了 ${ }^{[1]}$ 。那么首先，让我们来看看镁是怎么被发现的。”

张馆长首先请出了英国的戴维爵士(Humphry Davy), 正是他给镁这种元素命的名。随着阵阵掌 声, 戴维爵士的立体影像缓缓出现在同学们的面前。他向同学们热情地介绍道: “早在 1755 年, Joseph Black 先生就在石灰石中分出了一种叫苦土的东西, 换成科学的语言就是分离出了氧化钙中

收稿: 2020-03-22; 录用: 2020-03-31; 网络发表: 2020-05-06

“通讯作者, Email: yanjing@nwpu.edu.cn; yanyi@nwpu.edu.cn

基金资助: 西北工业大学 2020 年“课程思政”示范课程建设项目; 陕西省新工科研究与改革实践项目; 陕西省提升公众科学素质计划项目(2020); 西北工业大学实验核心课程建设(18GH0203014); 校高水平课程建设(19GH0101014); 2018 年度校级教育教学改革项目(W015304); 2019 年研究 生教育教学改革项目(19GA210101); 2019 学位与研究生教育研究基金(19GZ220101); 2019 年本科教学项目(19GH0101127, 19GH01001031, 19GZ1201) 
的氧化镁。在希腊一个叫做 Magnesium 的地方盛产这种苦土。我在发明了电解法制取金属元素后, 看到了有关苦土中这种未知的金属元素的文献, 顿时产生了极大的兴趣。1 808 年, 我成功地用电解 法制得了这种元素, 并将它命名为 Magnesium, 也就是大家口中的镁。”

听完戴维先生的讲解, 张馆长又接着向大家介绍: “镁的外层电子排布为 $1 s^{2} 2 s^{2} 2 p^{6} 3 s^{2}$, 和它的 好姐妹钻一样, 镁对最外层的两个电子也是无力看管, 经常搞买一送一的活动, 这也使得镁的还原 性比较强, 在金属活动性顺序里也是名列前茅, 与许许多多的元素都能 “自来熟” 地结合, 即使这 些元素不那么活泼也没关系。比如说氧化镁、碳化镁、氮化镁、硫化镁、氢化镁、硅化镁等化合物 就是镁与大家友谊的结晶。”

“而说到还原性，镁的燃烧现象就不得不提，”张馆长话毕，微笑着请出了今天的第二位嘉宾， 一位身着民国旗袍的优雅女士 $\mathrm{MgO}$ 小姐。她冲同学们微微一笑, 缓缓讲道: “镁是燃烧最明亮的金 属, 点燃后发出相当耀眼的白光。也正是如此, 在镁钙锶钡碱土四兄弟中, 只有镁这个小可怜没有 焰色反应。不过镁也相信 ‘天生我材必有用', 在烟花、照明弹及闪光弹中都担任了重要角色。除此 之外, 虽然镁的燃点只有 $500{ }^{\circ} \mathrm{C}$, 但燃烧温度可以高达 $3000{ }^{\circ} \mathrm{C}$, 因此常用作铝热反应的引发剂。 喜欢摄影的同学们肯定听说过 ‘镁光灯”。早在 19 世纪 60 年代, 氙灯还没有出现, 相机的闪光灯就 是通过点燃镁粉产生白光来实现的, 不过只能用一次, 使用起来也不安全, 现在已经被淘汰了。偷 偷告诉大家一个小秘密, 这镁光灯工作时又闪光又冒烟, 第一次见的时候, 可把我吓了一跳, 还以 为有妖怪要出现了呢。”

同学们哄堂大笑, 张馆长也忍俊不禁。告别了这位温柔的女士, 张馆长又向大家接着讲道: “根 据唯物主义辩证法, 事物皆是有两面性的, 镁也不例外。俗话常说水火无情, 大家想想镁着火后应 该怎么灭火呢? ”

同学们窃窃私语, 胆大的小明说: “当然用水啦。”

张馆长摇摇头说: “镁燃烧产生的高温会使其与水剧烈反应, 反应生成氢气无异于火上浇油。”

小王用手指着墙角红色的二氧化碳灭火器, 胆怯地说: “要不要试试这个灭火器呢? ”

还没等张馆长回答, 小强就说: “你忘了吗? 镁的性质很活泼, 她在二氧化碳里也可以燃烧啊!”

张馆长点点头说: “对, 二氧化碳和氧气一样, 都可以充当镁的助燃气体。同样, 镁也能在氮气 中欢快地燃烧。其实, 当着火不大时, 可以考虑用干燥的黄沙润熄。但是镁可以和二氧化硅反应生 成氧化镁和非晶硅, 虽然氧化硅耐高温, 可镁和硅又能反应生成 $\mathrm{Mg}_{2} \mathrm{Si}$, 这种物质在酸性条件下很 容易水解反应生成氢氧化镁和硅烷, 硅烷在空气中就能自燃冒出大量烟雾, 所以最好的办法是使用 $\mathrm{D}$ 类金属火灾专用 7150 灭火剂(偏硼酸三甲酯)。”

在同学们若有所思的目光中, 张馆长又微笑着请出了今天的第三位嘉宾一来自某军工研究所 的一位老工程师。他和蔼可亲地向同学们讲道: “在日常生活中, 很多地方都可以见到镁的身影, 比 如在炼钢时作为脱硫剂, 在制备钛、锆、铍、铀、铪时作为还原剂, 在生产球墨铸铁时作球化剂, 还有作为镁棒通过牺牲阳极的方式保护热水器等。不过今天, 我主要想和大家讲讲镁合金的应用, ” 说着，工程师打开了一个巨大的屏幕，屏幕的视频中出现了一位穿着军装的 “镁丽”女兵。

“大家好, 我是镁合金, 很高兴在这里遇见你们！正如你们所看到的, 我不仅容貌“镁”, 身材 也很好。作为一个女孩子, 体重管理很重要。所以我的密度只有铝先生的 $2 / 3$, 钢叔叔的 $1 / 4$, 和他 们这些大块头比起来, 我在军营中是非常轻巧的存在。” 说话间, 镁合金绽放了一个灿烂的笑容, 同 学们也感到非常亲切。“可是, 轻巧并不代表弱小, 咱们军营里的镁合金们, 那可个个都是帆国不让 须眉的存在。作为典型的轻质高强材料, 我们在比强度、弹性模量、抗冲击性能等力学性能上面和 其他金属材料相比可是不遑多让, 并且我们还有电磁屏蔽的功能, 所以也被用来制作飞机蒙皮、舱 体和壁板”。紧接着, 镁合金就举起了自己面前的哑铃, 完成了一系列复杂的健身动作, 并且阻截了 想要从她面前走过的电磁先生。这轻盈的身体里爆发出如此大的能量, 让在场的同学们都惊叹不已。

“此外, 我们还拥有其他很多士兵没有的柔韧性, ” 说着, 镁合金提升了自己周围的温度, 然后 
在高温的作用下, 很轻松地完成了下腰、一字马等高难度瑜伽动作。“我在高温下, 身体会变得非常

“柔软”, 能够很容易地变成很多复杂的形状, 所以, 我在军营中经常能扮演一些其他金属都很难完 成的角色, 比如说飞机发动机部件、直升机的传动箱和变速箱、导弹的支架等。并且, 我们也能很 快 “冷静' 下来, 这样的高温并不能在我们身上存在很久。” 话音刚落不久, 她的身体就由原来的通 红变成了银灰色。“因为这些优异的性能, 所以我也被用于歼击机的制造 ${ }^{[2,3]}$ 。值得一提的是, 我还 和碳纤维这个小后生合作过, 让哈勃望远镜飞上了太空！ [4]”

同学们听罢, 爆发出了响亮的掌声与欢呼声。此时, 老工程师向镁合金女兵敬了一个礼, 然后 接着说道: “感谢镁合金给大家做的介绍! 正如刚才大家所听到的, 镁合金在军事方面拥有非常大的 用处，而我国是稀土资源非常丰富的国家，在含稀土高耐热镁合金研究方面拥有独特优势。如何能 够好好地开发和利用这些资源，那就看你们的了!”

了解完镁合金的应用, 张馆长又开始介绍今天的第四位嘉宾—法国化学家维克多·格林尼亚先 生(Francois Auguste Victor Grignard), “1912 年, 41 岁的格林尼亚因为格氏试剂的发明获得了诺贝尔 化学奖。格氏试剂的出现丰富了烷基在有机合成中的角色, 使其不仅能作亲电试剂, 还可以作亲核 试剂。现在, 让我们用热烈的掌声, 欢迎格林尼亚先生为我们介绍这种重要的有机合成试剂!”

格林尼亚先生衣着考究, 带着 20 世纪欧洲上流社会知识分子的高贵气质。他亲切地向同学们讲 道: “在里昂大学学习期间, 我发现了镁的一类金属有机化合物, 通式为 $\mathrm{RMgX}$, 其中 $\mathrm{R}$ 为脂肪烃 基或芳香烃基, $\mathrm{X}$ 为卤素 $(\mathrm{Cl} 、 \mathrm{Br}$ 或 $\mathrm{I})$ 。在这种化合物中, 镁原子直接和碳相连, 由于极化作用, $\mathrm{C}-\mathrm{Mg}$ 键具有很高的反应活性, 是一种极强的路易斯碱, 这种试剂就是张馆长刚才所提到的格氏试剂。你 们现在常用卤代烃与镁粉在无水乙醚或四氢呋喃中反应制得, 制备过程必须在绝对无水、无二氧化 碳、无乙醇等具有活泼氢的物质(如: 水、醇、氨、卤化氢、末端炔等)条件下进行 ${ }^{[5]}$ 。

虽然格氏试剂的组成看似简单, 但是科学家们一直无法分析其三维结构, 具体的活性位点也因 其醚溶液中众多不同配位的镁元素而变得扑朔迷离, 进而也无法知道它的反应途径类型。虽然格氏 试剂早已应用在有机合成上多年, 但这个 “格氏试剂之迷” 一直争论不止, 直到挪威奥斯陆大学的 Odile Eisenstein 教授和 Michele Cascella 教授合作, 在美国化学会志(J.Am. Chem. Soc.)上近期发表了 一篇以 'The Grignard Reaction-Unraveling a Chemical Puzzle ${ }^{[6]}$ ' 为题的论文才为这场讨论画上了休 止符。可见格氏试剂这个 “简单”而又充满魅力的试剂是多么受到化学家们的喜爱。

话题回到格氏试剂的反应, 它可以发生多种反应, 主要包括: 烷基化反应, 羰基加成, 共轭加 成, 及卤代烃还原等, 尤其是烷基化醛和酮的反应, 仿佛一把充满魔力的钥匙, 打开了通向许许多 多结构新颖的有机化合物的崭新大门。不止这些, 它还可作为一个碱, 与醇、酚等反应生成醇盐; 可用于形成碳-杂原子键; 可与有机卤化物偶联……还有许多更惊奇的本领等待着科学家们去发掘! ”

讲完知识, 格林尼亚先生又向同学们勉励道: “我像你们这么大的时候, 还是个出了名的不学无 术、游手好闲的小混混。2 1 岁的时候, 波多丽女伯爵对我鄙夷的训斥一语惊醒梦中人。我痛定思痛, 离家出走, 努力弥补自己荒废多年的学业, 终于考入里昂大学。我想正是我的刻苦打动了我的恩师 巴比尔教授(Frederic Barbier), 他不但悉心教导我, 还把自己已经开创的课题交给我去研究, 这才有 了格氏试剂的发现。我曾想将这种试剂命名为格林尼亚-巴比尔试剂, 但教授却坚持认为这是我一个 人的功劳。他为人的淡泊和对知识的严谨公正是每一个科研人员应该具有的品质! 我也很庆幸我终 是没有幸负恩师对我的厚望。希望我的经历可以给今天的你们带来鼓励, 机会总是留给有准备的人, 但只要想努力, 何时都不会太晚。”

听了格林尼亚先生的经历和成就, 同学们不无惊喜。这时, 最后一位嘉宾出场了。她是某医学 研究所的一名研究员, 身穿白色的实验服, 坐在一个高脚登上, 抬手扶了扶眼镜, 向同学们介绍道: “接下来就让我们一起来认识一下这个神通广大的元素界 “大神” 吧! 在生物学医学方面, 镁的作 用极为重要。在生物机体中, 镁离子的含量也仅次于钙、钠、钾。人体内到处都有以镁为催化剂的 代谢系统，必须靠镁来进行的重要代谢至少有上百个。 
作为元素周期兄弟当中最低调的成员, 镁同志可以算得上是才华横溢的宝藏男孩啦。在这座生 物城池中他是起着中流砥柱作用的一员大将, 他平时会给业务繁忙的肌肉和神经打打下手, 帮助调 节肌肉的兴奋性, 有了镁的加入神经才可以保持镇定正常工作。镁的另一份工作是帮助调节神经递 质的分泌, 有了他的协助, 递质工厂才可以顺利产出血清素等优质产品供给; 同时镁还是城池中另 一项伟大工程的参与者, 他是几百种酶促反应的重要成员, 比如在蛋白质、碳水化合物以及脂肪的 消化, 蛋白质的合成, 核酸代谢, 细胞周期的调控以及激活多种 ATP 酶等过程中, 他都扮演着不可 或缺的角色。在细胞社区内, 镁又是责任重大的守门员, 掌管着钾、钠、钙离子细胞内外移动的 “通 道', 不仅可以维持生物膜电位, 还对膜的流动性也有一定的影响。

镁的涉猎极广，在医疗方面也逐渐得到重用。如果没有镁，生物城池就会面临糖尿病、高血压、 心血管疾病、偏头痛、痛经、免疫系统疾病、哮喘甚至抑郁症等危机。如果细胞社区缺少镁, 那么 镁的好兄弟钾也 “誓死” 不进入细胞, 进而导致生物城池无法维持正常的运作 ${ }^{[7]}$ 。而且在有敌军入 侵的时候, 比如在易引发老年痴呆的铝元素或是易引发不育的镉元素面前, 镁也会挺身而出与之殊 死搏斗, 英勇斩杀敌军, 恢复城池安宁。更为重要的是, 我国学者发现异甘草酸镁在新冠肺炎 (COVID-19)临床实践治疗中显示出良好疗效, 已被国家卫生健康委员会和国家中医药管理局纳入新 冠病毒肺炎管理规范 ${ }^{[8]}$ 。值得一提的是，镁及其合金在医用植入材料方面的贡献也是有目共睹的。 在生物城池某些部分受到严重破坏的时候, 镁当仁不让地承担起了重新建造的重担, 比起之前在任 的金属材料, 由镁参与建造的植入材料更加接近于原构造的密度和模量, 能够具有更好的生物相容 性。这里我要告诉大家, 镁合金的可降解材料的可行性已经在实验和临床上得到了一定的证实, 比 如用以做心血管支架以及骨固定植入物, 这意味着患者无需经历将植入材料取出时的二次伤害。但 是呢, 人无完人, 镁合金在生物城池内的存活寿命不够理想, 也就是说他的耐腐蚀性能并不是很好, 降解过程也有待进一步研究, 人们至少需要保证植入的材料在组织愈合完全之前不能过分降解, 所 以镁要想在医疗领域大显身手还需要更多的努力!

镁在植物中也是一名伟大的工程师, 如果缺少镁的存在, 植物就不能正常生长。当然镁最卓越 的功绩还是作为配位元素参与叶绿素的合成。生物圈中的生产者, 主要就是含有叶绿素的各种绿色 植物, 它们通过光合作用将光能转化成化学能固定下来。从这种意义上讲, 没有镁, 整个生物圈也 会土崩瓦解。所以大家现在能站在这里参观, 镁实在是功不可没!”

同学们向女研究员挥手告别, 今天的参观也接近了尾声。有同学问道: “既然是元素时空博物馆, 为什么没有未来呢? ”张馆长神秘一笑: “未来是现在创造的, 既绝密又不稳定, 镁的未来, 正掌握 在你们手中。天机不可泄露, 我只能告诉大家: 未来一片光明!”

\section{参 考 文 献}

[1] 高胜利, 杨奇. 化学元素新论. 北京: 科学出版社, 2019: 451-460.

[2] 姚灿, 郭芳琼. 装备制造技术, 2015, 42 (1), 122.

[3] 吴国华, 陈玉狮, 丁文江. 载人航天, 2016, 22 (3), 281.

[4] 李中权, 肖旅, 李宝辉, 王先飞. 上海航天, 2019, 36(2), 9 .

[5] 焦雷. 大学化学, 2004, 19 (5), 57.

[6] Peltzer, R. M.; Gauss, J.; Eisenstein, O.; Cascella, M. J. Am. Chem. Soc. 2020, 142 (6), 2984.

[7] 赵霖, 鲍善芬. 科技导报, 1992, 10 (11), 30 .

[8] 正大天晴异甘草酸镁被纳入新冠病毒肺炎管理规范. [2020-03-21]. https://www.cttq.com/news/578863.htm. 